\title{
Photorefractive Amplification of Few-cycle Laser Pulses
}

\author{
Hajime NISHIOKA, Shuji OHTA, and Hitoshi TOMITA \\ Institute for Laser Science, University of Electro-Communications, \\ 1-5-1 Chofugaoka, Chofu, Tokyo 182-8585
}

(Received January 17, 2008)

\begin{abstract}
Few-cycle pulse amplification by two-photon arranged photorefractive device has been demonstrated. An extraction efficiency of $27 \%$ has been achieved with a gain-bandwidth of $32 \mathrm{THz}$.
\end{abstract}

Key Words: Ultrashort pulse lasers, Multi-photon interference, Photorefractive effect

\section{Introduction}

Coherent combing the output beam from multiple lasers is an efficient approach for scaling the brightness of laser system. Recently progress has been made with $\mathrm{CW}$ or quasi-CW lasers using wavelength multiplexing or stationary phase-locked technique. In femtosecond time scale, coherent combining with wavelength multiplexing effective to decrease pulse duration and to increase brightness but additional requirements are arisen; group-delay and group-delay-dispersion between the laser beams have to be compensated.

In this paper, we demonstrate few-cycle pulse amplification based on the coherent beam combining by two-photon induced photorefractive effect. This scheme is schematically shown in Fig. 1. A femtosecond seed pulse and a chirped pump pulse interact in a PR medium that has perfect transmittance at the laser wavelength and has two-photon absorption. The dynamic two-photon interaction between the seed pulse and the pump pulse form a chirped index grating as a second order cross-correlation function ${ }^{1)}$. The spatial phase structure of the two-photon arranged grating has the same phase structure of the chirped pulse. The first order diffraction of the pump beam appears in the same direction to the seed. In photorefractive materials that operate by pure diffusion, in where the phase shift between the light fringe and the PR index grating is $\pi / 2$, no phase coupling is produced between the seed and the pump beams. Thus the seed pulse is amplified in the medium and its phase structure is kept constant regardless of the energy transfer. In the other words, phase modulation or distortion of the pump beam is disappeared in the output. The self-cancellation of phase information or distortion of the pump beam is actually equivalent to the regeneration of the seed pulse i.e. coherent amplification/beam combining of femtosecond light pulses based on the frequency-domain phase conjugation ${ }^{1)}$.

\section{Experiments}

A Fe doped $\mathrm{LiNbO}_{3}$ crystal was selected for the first demonstration because it has broad absorption spectrum around two-photon wavelength and has the IR transmission band. A 9 fs mode-lock Ti: $\mathrm{Al}_{2} \mathrm{O}_{3}$ laser operating at $800 \mathrm{~nm}$ with a spectra width of $\lambda=115 \mathrm{~nm}$ (FWHM), and a repetition rate of $76 \mathrm{MHz}$ was used as the seed pulse. A chirped pump pulse having a pulse duration of $400 \mathrm{fs}$ (FWHM) was generated by a $40 \mathrm{~mm}$-thick quartz block

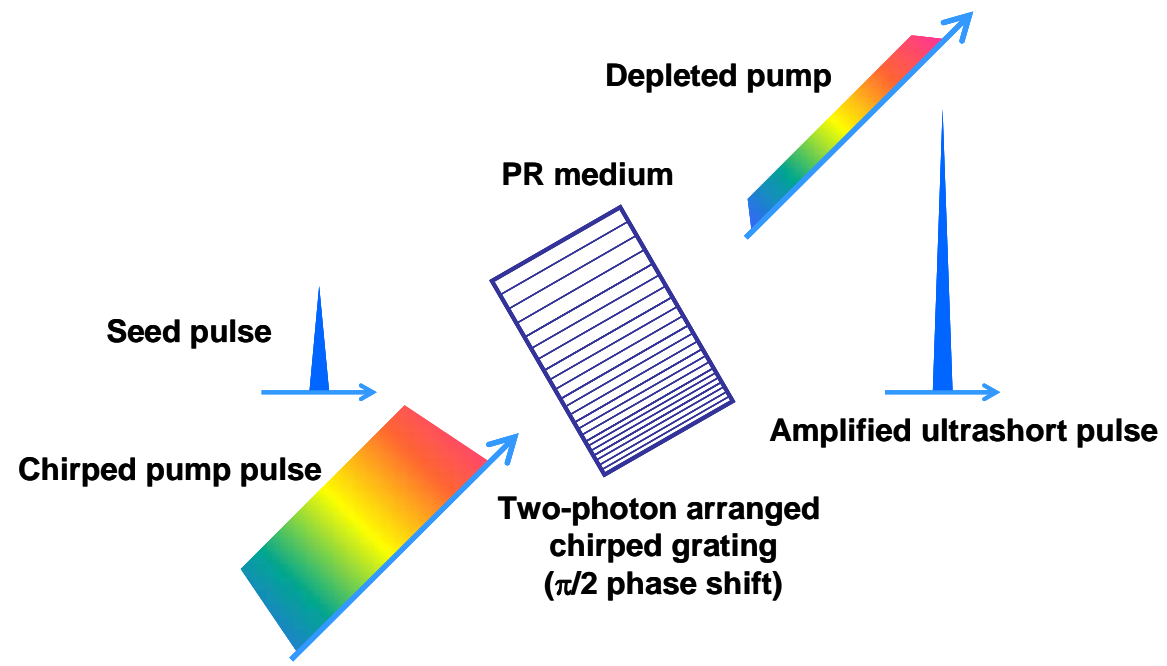

Fig.1. Schematic illustration of the two-photon arranged photo-refractive amplifier/beam combiner: TAPA. Spatially chirped grating is formed in the TAPA as a second order cross correlation function between the seed and pump pulses. The first order diffraction of the pump pulse has the same phase structure of the seed. The unidirectional energy flow, the pump to seed, is produced by the $\pi / 2$ phase-shift via PR effect. 
stretcher. The seed pulse interacted with the chirped pump pulse in the $1 \mathrm{~cm}$-thick crystal with a crossing angle of $\mathrm{F} / 5$. The grating wave vector was parallel to the c-axis.

Figure 2 is showing diffraction efficiency of two-photon arranged grating by the pump beam having $5.1 \mathrm{MW} / \mathrm{cm}^{2}$ (peak intensity) as a function of time. These spectra were measured accumulatively after every $30 \mathrm{~s}$ exposure to the pump and seed pulses. The extraction efficiency from the chirped pump to output was $27 \%$. A gain bandwidth was measured to be $69 \mathrm{~nm}$ $(=32 \mathrm{THz})^{2}$.

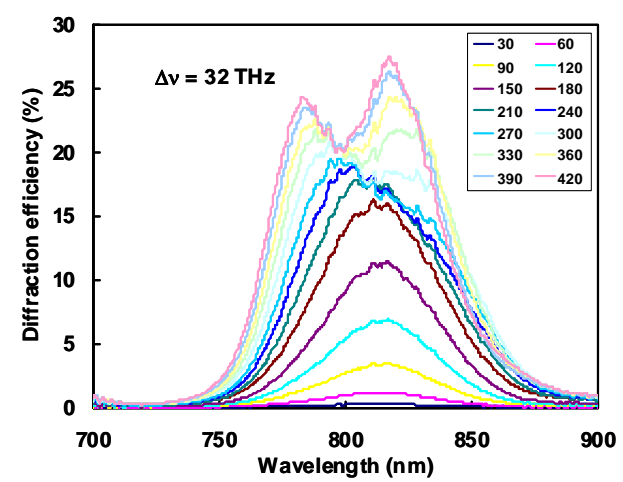

Fig.2. Bandwidth of the two-photon arranged photorefractive beam combiner. Each spectrum is taken every $30 \mathrm{~s}$ exposure up to $420 \mathrm{~s}$.

The response time decrease with increase of average light intensity so that time-dependent dynamic phase correction is possible. We have attenuated seed pulse energy by a factor of 8 to measure Fourier phase as a function of the gain growth. The phase difference between the amplified output and the seed as a function of exposure time measured by the SPIDER is shown in Fig. 3. The Fourier phase was kept constant regardless of the saturated amplification. The reverse energy flow (i.e. the seed to the pump) has been observed when we reversed c-axis. The $\mathrm{LiNbO}_{3}$ crystal doesn't operate with pure diffusion; however, effectively coherent amplification was possible based on the Fourier-phase-conjugation.

\section{Conclusion}

Few-cycle pulse amplification by the two-photon induced photorefractive effect with a bandwidth of $32 \mathrm{THz}$ has been demonstrated. The group-delay and group-delay-dispersion mismatches the seed and pump beam have been automatically compensated. This scheme can be extended to multi-pump-beam configuration for wavelength and/or angular multiplexing to combine the multi-pump beams in the same single-chip device.

Part of this work, the frequency-domain-phase correction scheme and the two-photon arranged photorefractive amplification, has been reported in Ref. 1), and 2), respectively. This work is supported by a by a grant-in-aid for scientific research, and the 21st Century Center of Excellence (COE) program from the Ministry of Education, Culture, Science, Sports and Technology.

\section{References}

1) H. Nishioka, H. Tomita, K. Hayasaka, and K. Ueda: Opt. Exp. 14 (2006) 7447

2) H. Nishioka, K. Hayasaka, S. Ohta, H. Tomita, and K. Ueda: Opt. Exp. 15 (2007) 5018.
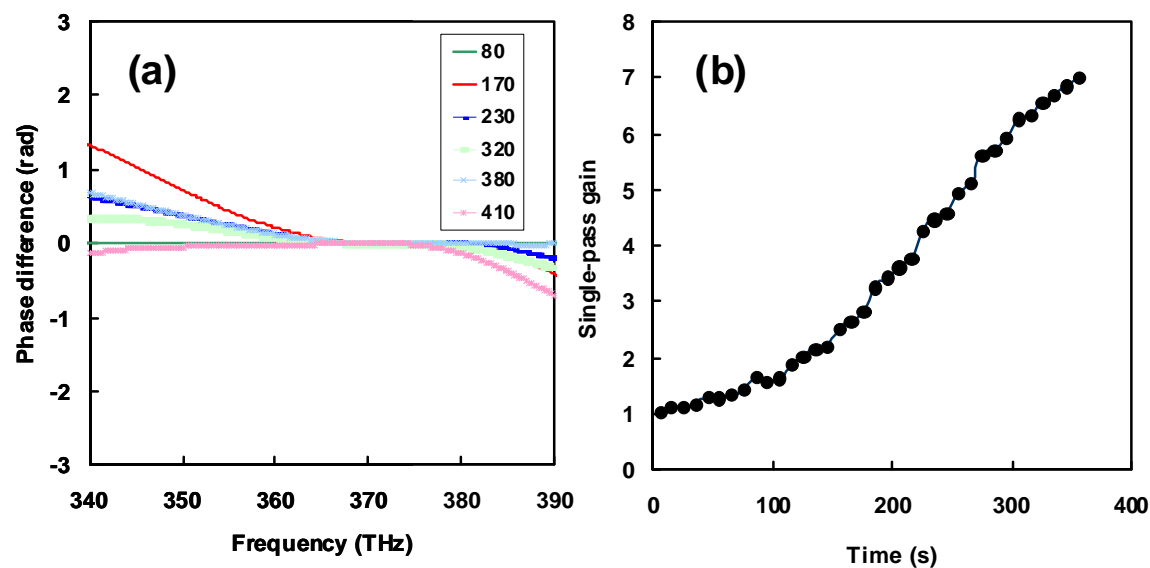

Fig.3. Fourier phase (a) and gain in brightness (b) as a function of exposure time. The parameter in (a) is the exposure time in second. The group-delay-dispersion in the PR crystal itself has been subtracted in this figure. The energy ratio of the pump and seed pulses is $8: 1$. 